\title{
Positron Lymphography: Multimodal, High-Resolution, Dynamic Mapping and Resection of Lymph Nodes After Intradermal Injection of ${ }^{18}$ F-FDG
}

\author{
Daniel L.J. Thorek ${ }^{1}$, Diane S. Abou ${ }^{2}$, Bradley J. Beattie ${ }^{3}$, Rachel M. Bartlett ${ }^{3}$, Ruimin Huang ${ }^{1}$, \\ Pat B. Zanzonico ${ }^{3}$, and Jan Grimm ${ }^{1,2}$ \\ ${ }^{1}$ Department of Radiology, Memorial Sloan-Kettering Cancer Center, New York, New York; ${ }^{2}$ Program in Molecular Pharmacology \\ and Chemistry, Memorial Sloan-Kettering Cancer Center, New York, New York; and ${ }^{3}$ Department of Medical Physics, Memorial \\ Sloan-Kettering Cancer Center, New York, New York
}

The lymphatic system plays a critical role in the maintenance of healthy tissues. Its function is an important indicator of the presence and extent of disease. In oncology, metastatic spread to local lymph nodes (LNs) is a strong predictor of poor outcome. Clinical methods for the visualization of LNs involve regional injection and tracking of ${ }^{99 m}$ Tc-sulfur colloid ( ${ }^{99 m} \mathrm{Tc}-\mathrm{SC}$ ) along with absorbent dyes. Intraoperatively, these techniques suffer from the requirement of administration of multiple contrast media ( ${ }^{99 \mathrm{~m} T c-S C}$ and isosulfan blue), unwieldy $\gamma$-probes, and a short effective surgical window for dyes. Preclinically, imaging of transport through the lymphatics is further hindered by the resolution of lymphoscintigraphy and SPECT. We investigated multimodal imaging in animal models using intradermal administration of ${ }^{18} \mathrm{~F}-\mathrm{FDG}$ for combined diagnostic and intraoperative use. PET visualizes LNs with high sensitivity and resolution and low background. Cerenkov radiation (CR) from ${ }^{18} \mathrm{~F}-\mathrm{FDG}$ was evaluated to optically guide surgical resection of LNs. Methods: Imaging of ${ }^{18} \mathrm{~F}-\mathrm{FDG}$ uptake used PET and sensitive luminescent imaging equipment (for CR). Dynamic PET was performed in both sexes and multiple strains (NCr Nude, $\mathrm{C} 57 \mathrm{BL} / 6$, and $\mathrm{Nu} / \mathrm{Nu}$ ) of mice. Biodistribution confirmed the up-

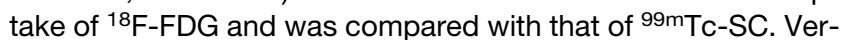
ification of uptake and the ability to use ${ }^{18} \mathrm{~F}-\mathrm{FDG}$ CR to guide nodal removal were confirmed histologically. Results: Intradermal injection of ${ }^{18} \mathrm{~F}-\mathrm{FDG}$ clearly revealed lymphatic vessels and LNs by PET. Dynamic imaging revealed rapid and sustained labeling of these structures. Biodistribution of the radiotracer confirmed the active transport of radioglucose in the lymphatics to the local LNs and over time into the general circulation. ${ }^{18} \mathrm{~F}-\mathrm{FDG}$ also enabled visualization of LNs through CR, even before surgically revealing the site, and guided LN resection. Conclusion: Intradermal ${ }^{18} \mathrm{~F}-\mathrm{FDG}$ can enhance the preclinical investigation of the lymphatics through dynamic, high-resolution, and quantitative tomographic imaging. Clinically, combined PET/Cerenkov imaging has significant potential as a single-dose, dual-modality tracer for diagnostics (PET/CT) and guided resection of LNs (Cerenkov optical).

\footnotetext{
Received Feb. 13, 2012; revision accepted May 9, 2012.

For correspondence or reprints contact: Jan Grimm, 1275 York St., Box 248, Memorial Sloan-Kettering Cancer Center, New York, NY 10065.

E-mail: grimmj@mskcc.org

Published online Jul. 7, 2012.

COPYRIGHT (C 2012 by the Society of Nuclear Medicine and Molecular Imaging, Inc.
}

Key Words: lymph node mapping; PET/CT; Cerenkov; intraoperative

J Nucl Med 2012; 53:1438-1445

DOI: 10.2967/jnumed.112.104349

$\mathbf{T}$ he lymphatic system is a complex network of capillaries that collect and drain the interstitial fluid and macromolecules that extravasate at the intersection of the arterial and venous vasculature (1). Lymph nodes (LNs) are interspersed throughout this network to filter the fluid and provide a site for immunoregulatory lymphocytes to encounter antigen. Because LNs are important foci of the immune system, their status is an important indicator of many diseases (2).

In oncology, metastatic cells have the capacity to spread through the lymphatic system. The sentinel LN hypothesis states that the closest draining $\mathrm{LN}$ to a tumor (the sentinel node) can be evaluated for tumor cell invasion and provide information on the extent of disease. LN involvement is a crucial prognostic marker for melanoma $(3,4)$ and for cancers of the prostate (5), cervix $(6,7)$, and breast (8). Sentinel $\mathrm{LN}$ involvement is the most important prognostic indicator for patients with primary breast cancer $(9,10)$. General clinical practice is to remove these LNs and analyze them for the presence of tumor cells.

Lymphatic mapping enables visualization of local LNs to guide sentinel $\mathrm{LN}$ resection in the clinical setting. It is also useful in the research space to study the biologic roles of the lymphatics. Tools for mapping have included MRI (11), scintigraphic $(12,13)$ and tomographic nuclear imaging (14), and optically absorbing (15) and fluorescent dyes (16). This array of molecular modalities possesses a wide range of sensitivities, depths of penetration, resolution, throughput, and cost (17).

Clinically, LNs are localized through injection of ${ }^{99 \mathrm{~m}} \mathrm{Tc}-$ labeled sulfur colloid ( $\left.{ }^{99 \mathrm{~m}} \mathrm{Tc}-\mathrm{SC}\right)$, often with the subsequent injection of isosulfan blue dye to guide resection. The technique affords a high identification rate (8). However, there is limited specificity and transport of the colloid, providing only 
2-dimensional planar information. Tomographic imaging (SPECT) is possible, but it requires long imaging times and has limited resolution. The vital dye may also travel past the sentinel LN. In addition to questions concerning the global supply of ${ }^{99} \mathrm{Mo}$ for generators, these limitations have spurred development of replacement (18) or alternative contrast agents exploiting various modalities $(3,19)$.

Here, we demonstrate dual-modality imaging using a single tracer for both lymphatic mapping and guided surgical resection with the ubiquitous PET radiotracer ${ }^{18} \mathrm{~F}-\mathrm{FDG}$. In place of $\mathrm{LN}$ accumulation of ${ }^{18} \mathrm{~F}$-FDG after systemic delivery, injection is performed directly into the rich lymphatic bed of the

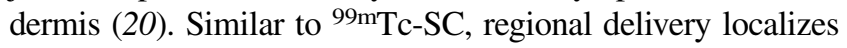
transport of ${ }^{18} \mathrm{~F}-\mathrm{FDG}$ through the lymphatic vessels to draining nodes. ${ }^{18} \mathrm{~F}$-FDG PET/CT clearly delineates the fine vessels and LNs in rodents.

In addition to the low background, inherently quantitative nature, high sensitivity, and high temporal resolution of PET lymphography, the radiotracer itself enables optical guidance to localize nodes through emission of Cerenkov radiation (CR). Produced by high-energy charged particles emitted on radioactive decay $\left(\beta^{+}\right.$for $\left.{ }^{18} \mathrm{~F}\right)$, the visible-wavelength $\mathrm{CR}$ photons from PET isotopes can be acquired using highly sensitive camera equipment $(21,22)$. PET lymphography also allows white-light imaging (standard photography) of the subject, providing anatomic localization for surgical investigations (23). Thus, through a single intradermal injection of ${ }^{18} \mathrm{~F}-\mathrm{FDG}$, a clinically approved and widely available agent, we achieved both exquisite nuclear tomographic lymphatic mapping for preclinical or preoperative investigation and an optical signal to lead intraoperative resection.

\section{MATERIALS AND METHODS}

\section{Materials}

Isosulfan blue (Lymphazurin) was acquired from Tyco Healthcare Group LP. ${ }^{18} \mathrm{~F}-\mathrm{FDG}$, with a specific activity of more than 41 $\mathrm{MBq} / \mu \mathrm{mol}(>11 \mathrm{mCi} / \mu \mathrm{mol})$, was supplied by IBA Molecular, and ${ }^{99 \mathrm{~m}} \mathrm{Tc}-\mathrm{SC}$ was from Cardinal Healthcare. Both tracers had a radiochemical purity greater than $98 \%$. Tracers were acquired through the Radiopharmacy of the Nuclear Medicine Service at Memorial Sloan-Kettering Cancer Center.

\section{Animal Experiments}

Animal experiments were in accordance with guidelines from the Institutional Animal Care and Use Committee and with the Guide for the Care and Use of Laboratory Animals from the National Institutes of Health (NIH) (24). All animal procedures were performed under anesthesia by inhalation of a $1 \%-2 \%$ isoflurane-air mixture (Baxter Healthcare). Animals were obtained from either Harlan Laboratories (nu/nu and C57BL/6) or Charles River Laboratories (NCr nude). Male nu/nu mice (12 wk) were used for the representative PET/CT figures, female nu/nu mice (12 wk) for the CR imaging, and male C57BL/6 mice (6-8 wk) for acute biodistribution studies.

\section{Intradermal Injections}

${ }^{18} \mathrm{~F}$-FDG and ${ }^{99 \mathrm{~m}} \mathrm{Tc}-\mathrm{SC}$ were injected intradermally to study the biodistribution and imaging of the lymphatic system. Hypo- dermic needles $(30$-gauge $\times 1.9 \mathrm{~cm}$ [0.75 in], Kendall Monoject; Tyco Healthcare) were used. Dorsal tail injections $(\sim 1.2 \mathrm{MBq}$ of ${ }^{18} \mathrm{~F}-\mathrm{FDG}$ or $500 \mathrm{kBq}$ of ${ }^{99 \mathrm{~m}} \mathrm{Tc}-\mathrm{SC}$ in a volume of $\left.25-35 \mu \mathrm{L}\right)$, the bevel of the needle directly in the dermis, were made at approximately $30 \mathrm{~mm}$ from the base of the tail. Constant pressure was applied over $30 \mathrm{~s}$, with care not to disrupt the deep lymphatics or lateral veins.

\section{Small-Animal PET and PET/CT}

Mice were imaged prone, using the Focus 120 microPET smallanimal scanner (Concorde Microsystems Inc.), with an energy window of 350-700 keV and coincidence-timing window of $6 \mathrm{~ns}$. Acquisition began immediately after intradermal or (for intravenous comparison) retroorbital injection for as long as $60 \mathrm{~min}$.

A custom-built restraint device registered PET and CT image data (25). CT was performed using a microCAT II (ImTek Inc.) scanner operating at $60 \mathrm{kVp}$ and $0.8 \mathrm{~mA}$, with 2-mm aluminum filtration. The fusion montage was arranged in ImageJ (NIH). Three-dimensional rendering was performed using Amira (version 5.0; Visage Imaging $\mathrm{GmbH}$ ).

PET data were corrected for detector nonuniformity, dead time, random coincidences, and physical decay. List-mode data were binned into histograms at durations of $0.5,1$, and 5 min to study different features of ${ }^{18} \mathrm{~F}$-FDG transport and uptake. Images were reconstructed by both maximum a priori and 3-dimensional filtered backprojection using a ramp filter with a cutoff frequency equal to the Nyquist frequency into a $128 \times 128 \times 95$ matrix $(26)$. Images were analyzed using ASIPro (Concorde Microsystems Inc.), with window and level settings adjusted for optimal visualization of LNs. To assess flow rates, volumes of interest were drawn around LNs, and time-activity curves were generated.

\section{CR Optical Imaging}

After sacrifice, CR images for guided resection were obtained using the IVIS 200 (Caliper Life Sciences). This system uses a cryo-cooled charge-coupled device for high-sensitivity detection of low-intensity sources. Two-minute postmortem exposures were used to identify the presence and distribution of CR from ${ }^{18} \mathrm{~F}-\mathrm{FDG}$ in mice. Images were taken after each stage: removal of the dorsal skin, surgical incision to reveal the sacral nodes, and removal of the nodes. Excised tissues were then imaged. To bias a single side for LN draining and provide an internal contralateral reference (of little or no activity), lateral dermal tail injections were made. To visualize distribution differences by route of administration, a separate cohort was given an equal retroorbital dose $(\sim 1.2 \mathrm{MBq})$ of ${ }^{18} \mathrm{~F}-\mathrm{FDG}$, followed by PET/CT and optical imaging $(n=4)$.

\section{Biodistribution Studies}

We intradermally injected anesthetized mice with a 3:1 (volume) mixture of ${ }^{18} \mathrm{~F}-\mathrm{FDG}$ (or ${ }^{99 \mathrm{~m} T c-S C}$ ) and isosulfan blue. Animals were awakened and at the given endpoints sacrificed (10 or $60 \min , n=5$ ). Major organs and LNs (including the sacral, inguinal, axial, and caudal nodes) were excised, washed, and wicked of excess water. Background-corrected count-per-minute data of the excised tissues were acquired on an automatic Wizard $\gamma$-counter (Perkin Elmer) using an energy window of 400-600 $\mathrm{keV}$ for ${ }^{18} \mathrm{~F}$ and ${ }^{84}-163 \mathrm{keV}$ for ${ }^{99 \mathrm{~m}} \mathrm{Tc}$. Counts per minute were converted to becquerels using a standard calibration curve, decaycorrected to the time of injection, and reported as percentage of injected dose (\%ID) and normalized to mass in grams of tissue (\% ID/g). 
The weights of many of the LNs in the mouse are on the order of only a few milligrams. As such, special care was taken to obtain accurate weights for in vivo biodistribution studies. An analytic balance (accurate to 1/100th of a milligram) was used for all tissues (Excellence Plus XP; Mettler Toledo). Rather than standard $(12 \times 75 \mathrm{~mm})$ tubes, 2.0-mL Safe-Lock Eppendorf tubes (Eppendorf North America) were used to minimize dehydration of tissues and minimize weight variation. Individual empty tubes were weighed before necropsy, and all tissues were weighed immediately after $\gamma$-counting. To further reduce weighing error, contralateral nodes were pooled. The weights of tissues are included in the supplemental data (Supplemental Fig. 1; available online only at http:// jnm.snmjournals.org).

\section{Immunohistochemistry}

CR signal was used to guide excision of suspected sacral LNs. In addition to LNs, adjacent (non-Cerenkov luminescent) but visually indistinguishable tissue was excised. Sections $(5 \mu \mathrm{m})$ were stained for podoplanin and counterstained with hematoxylin. Detailed immunohistologic procedures are included in the supplemental data.

\section{RESULTS}

\section{Tracer Selection}

Before experimentation with small-molecule radiotracers for multimodal lymphatic imaging, it was necessary to choose a suitable probe. ${ }^{18} \mathrm{~F}$-based tracers have favorable positron emission characteristics for annihilation photon imaging and also produce CR for optical imaging. There is a wide choice of fluorinated compounds, including $\mathrm{Na}^{18} \mathrm{~F},{ }^{18} \mathrm{~F}$-fluoro-3'-deoxy-3'-L-fluorothymidine, and ${ }^{18} \mathrm{~F}$ fluorocholine. However, ${ }^{18} \mathrm{~F}-\mathrm{FDG}$ is the most widely used PET tracer and also the most widely available. In addition to being well tolerated, ${ }^{18} \mathrm{~F}$-FDG can be produced with high specific activity, is routinely available at many sites, and will not interfere with histologic evaluation (staining) of tissue. In tissue, ${ }^{18} \mathrm{~F}$ produces on the order of 14 Cerenkov photons per disintegration, making in vivo CR imaging facile at diagnostic doses (27).

\section{F-FDG PET/CT}

PET images demonstrate that the lymphatic vessels and nodes can be visualized after the intradermal administration of ${ }^{18}$ F-FDG (Fig. 1). Radiotracer distribution and imaging studies were performed on mice after intradermal injection into the tail. The injection site, several centimeters from the base of the tail, drains through lymphatic vessels and nodes that lie medially deep within the animal. This location was chosen on the basis of the tail excision model as described by Boardman et al. (28) and its use for recent lymphoscintigraphic work $(29,30)$.

The combination of PET for high-sensitivity detection of the exogenous tracer and CT for boney structures provides accurate and detailed localization of lymphatic structures. LNs and lymphatic vessels can be clearly identified from a 1 -min acquisition at $10 \mathrm{~min}$ after injection (Fig. 1).

Initially, the radiotracer is drained from the extracellular space at the injection site through the vessels rostrally. Draining through both lateral lymphatic vessels can be clearly seen on PET images. Flow and retention demarcate the sacral nodes. Further lymphatic draining then leads to distinction of first the caudal and then the mesenteric nodes. All structures are clearly visible, with minimal background signal, because the ${ }^{18} \mathrm{~F}-\mathrm{FDG}$ at this time point has not diffused or been drained into the vascular system.

\section{Dynamic Imaging}

Dynamic imaging using this technique can reveal features of the lymphatic drainage and presence of the nodes and be accomplished over an extended period (Fig. 2). The general distribution of the ${ }^{18} \mathrm{~F}-\mathrm{FDG}$ initially follows that described above. Over time, the amount of ${ }^{18}$ F-FDG transported along with lymph into the vascular system, and the likely direct diffusion of the tracer into blood vessels, increases the background activity. Thus, at $60 \mathrm{~min}$, the kidneys, bladder, liver, and heart were often visible.
FIGURE 1. Three-dimensionally rendered positron lymphography images demonstrate high-resolution mapping using intradermal administration of ${ }^{18} \mathrm{~F}-\mathrm{FDG}$ to a male $\mathrm{nu} / \mathrm{nu}$ mouse at multiple angles $(\mathrm{A}-\mathrm{C})$. Radiotracer is transported from site of injection through lymphatic system, enabling visualization of lymph flow. Delineation of LN draining injection site is clearly distinguished $(B$; insert is without fused CT). At 10 min after injection, we can identify lymphatic vessels in tail leading first to sacral nodes (1). Tracer then moves proximally to caudal (2) and then mesenteric nodes (3). Gradient arrow indicates direction of flow. Three-dimensionally rendered PET data are generated from weighted average of intensities from all 2-dimensional slices. As such, it is strictly semiquantitative; color bar indicates range of intensities. Max = maximum; Min $=$ minimum.
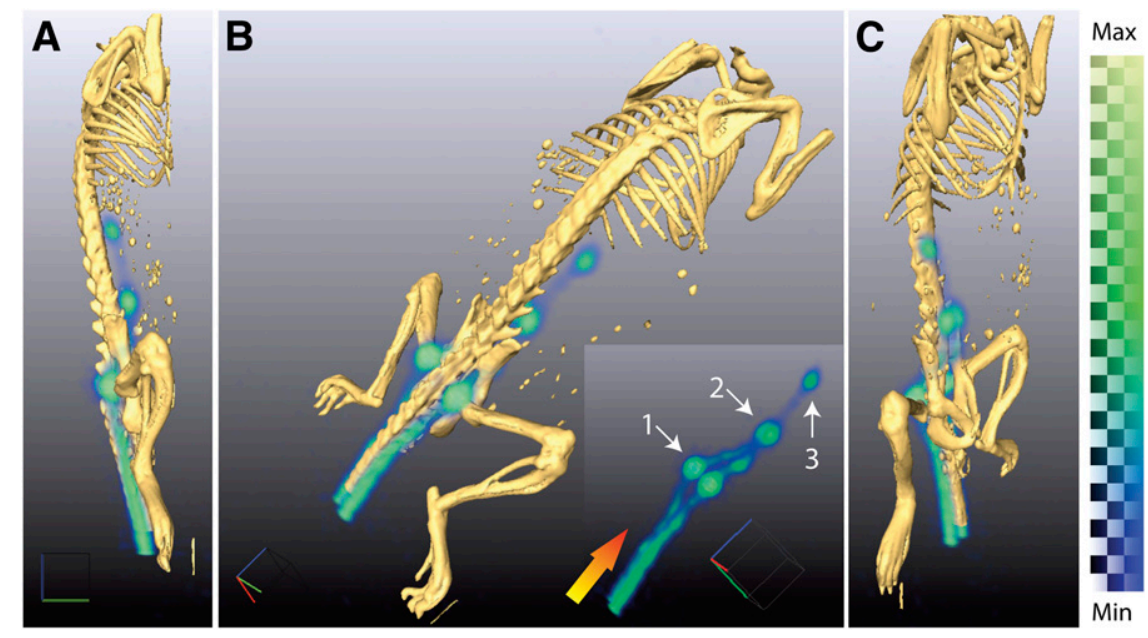

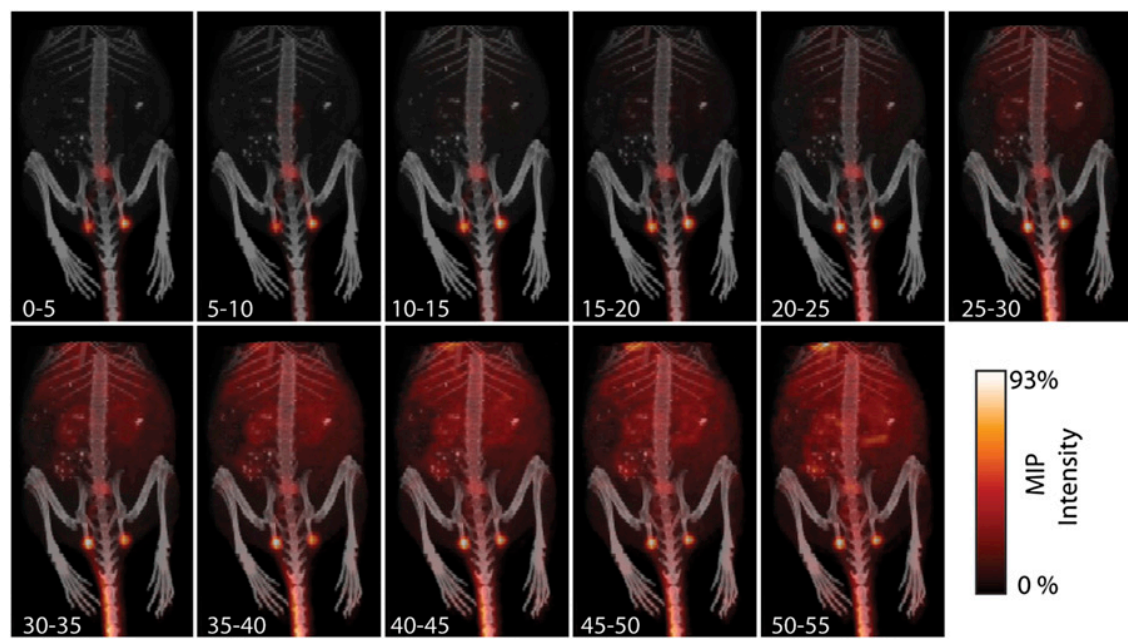

FIGURE 2. Dynamic imaging of progress of lymphatic transport of small-molecule radiotracer (5-min intervals). Within minutes, ${ }^{18} \mathrm{~F}-\mathrm{FDG}$ has begun to be cleared from injection site through lymphatic system. Transport through and uptake at LN makes these features obviously apparent. Background tissue uptake of tracer builds slowly over approximately 30-35 min, via lymphatic clearance and diffusion of the small molecule. However, clearly identifiable presence of nodes even at late time points is significant, enabling ready identification of these nodes at $1 \mathrm{~h}$ after injection. Detailed imaging of transport immediately after injection is shown in Figure 3. MIP = maximumintensity projection.
Imaging frame rates were sufficiently short (Fig. 3A) to reveal the kinetics of initial radiotracer distribution. This high temporal resolution is combined with quantitative readout of tracer concentration. In addition, the high-frame-rate dynamic and high-resolution lymphography can be given anatomic context through fusion with a CT (or MRI) acquisition. The inherently quantitative information from PET allows for the assessment of lymphatic function through tracer distribution. Over the linear portion of their accumulation, these healthy LNs demonstrate approximately 5.7-6.5 $\mathrm{Bq} / \mathrm{s}$ transport of ${ }^{18} \mathrm{~F}-\mathrm{FDG}$, whereas the most distant mesenteric node displays a lower rate of $1.17 \mathrm{~Bq} / \mathrm{s}$ of ${ }^{18} \mathrm{~F}-\mathrm{FDG}$ (Fig. 3B).

\section{Acute Biodistribution}

We determined the distribution of the locally administered ${ }^{18} \mathrm{~F}-\mathrm{FDG}$ at 2 time points, 10 and $60 \mathrm{~min}$ after injection. The biodistribution recapitulates the image data and is presented as both \% ID (to convey gross tracer distribution) and \% ID/g (to communicate image contrast) (Fig. 4).

Initially, at $10 \mathrm{~min}$, most of the dose is still present at the injection site in the tail (Fig. 4A). Small amounts are present in the blood (mean \pm SEM, $0.57 \pm 0.21 \%$ ID) and major organs such as the liver $(0.98 \pm 0.08 \%$ ID). After $1 \mathrm{~h}$, approximately $28.5 \pm 8.17 \%$ ID remains in the tail, with a significant amount in the bladder $(2.79 \pm 1.84 \% \mathrm{ID})$ and active muscle (heart, $1.08 \pm 0.20 \% \mathrm{ID}$ ). At $10 \mathrm{~min}$, the values in the injection site's draining sacral $(0.11 \pm 0.02 \% \mathrm{ID})$ and caudal nodes $(0.09 \pm 0.01 \%$ ID) are an order of magnitude less than those in the larger organs.

The localization of ${ }^{99 \mathrm{~m}} \mathrm{Tc}-\mathrm{SC}$ to sentinel LNs is exceptional as well (Supplemental Fig. 3). However, in comparison to ${ }^{18} \mathrm{~F}-\mathrm{FDG}$, the ${ }^{99 \mathrm{~m}} \mathrm{Tc}-\mathrm{SC}$ moves more slowly from the injection site. At 10 and $60 \mathrm{~min}$ after injection, $80.79 \pm$ 1.52 and $79.83 \pm 0.65 \%$ ID remain in the tail, respectively. This reflects the fact that little of the colloidal tracer is able to drain from the injection site. For ${ }^{18} \mathrm{~F}-\mathrm{FDG}$, at 10 and $60 \mathrm{~min}$, these values are $43.85 \pm 0.92$ and $28.59 \pm 8.17 \% \mathrm{ID}$, respectively. Here, clearance occurs through processes that include lymph transport and diffusion of the small molecule. The ${ }^{99 \mathrm{~m}} \mathrm{Tc}-\mathrm{SC}$ particles also localize more slowly to the nodes; an increase in \%ID is seen from 10 to $60 \mathrm{~min}$, the opposite of the case for ${ }^{18} \mathrm{~F}-\mathrm{FDG}$. This timing is concordant with the significantly larger diameter and mass of the colloid, favoring faster transport of ${ }^{18} \mathrm{~F}-\mathrm{FDG}$.

After normalization by the mass of the tissues to yield $\%$ ID/g values, the high node-to-other tissue values replicate the clear imaging delineation of the LNs (Fig. 4B). Active transport of the radiotracer from the dermal tail injection site through the lymphatic vessels and to the draining nodes presents highly specific anatomic localization of the tracer. The sacral nodes exhibit $183 \pm 79.0 \% \mathrm{ID} / \mathrm{g}$ (mean \pm SEM) at $10 \mathrm{~min}$ after injection. Nodes that are not in the draining pathway of the tail injection site (e.g., the axillary nodes $[0.77 \pm 0.392 \% \mathrm{ID} / \mathrm{g}]$ ) contain activity that is equivalent to the body background.

The exquisite delineation of the nodes from the PET acquisitions is a result of the high node-to-muscle and node-toblood ratios (Fig. 5). These compartments commonly confound systemic ${ }^{18} \mathrm{~F}-\mathrm{FDG}$ imaging, particularly in attempts to image lymphatic structures at early time points. However, the anatomic specificity of the intradermal injection leads to localization of the tracer in the draining nodes, with minimal blood or muscle extravasation. The ratio of $\mathrm{LN}$ to muscle decreases with time but is still high at $60 \mathrm{~min}$, and the LN-toblood ratio increases as free ${ }^{18} \mathrm{~F}-\mathrm{FDG}$ is excreted. This finding is significant because the biodistribution studies were performed on animals that were not anesthetized; therefore, no effort was made to limit metabolic uptake of the tracer in active muscles. No contrast enhancement is seen in LNs that are not in the drainage path from the injection site (e.g., axillary lymphatics).

\section{Cerenkov-Guided Intraoperative Imaging}

The transit and accumulation of the ${ }^{18} \mathrm{~F}-\mathrm{FDG}$ at the sacral nodes provides sufficient $\mathrm{CR}$ to guide surgical intervention and resection of LNs (Fig. 6). The intradermal injection isolates the ${ }^{18} \mathrm{~F}-\mathrm{FDG}$ to the draining lymphatic channels and their associated LNs. The sacral node can be seen after removal of the skin (i.e., in an intraoperative setting), before any 
Axial
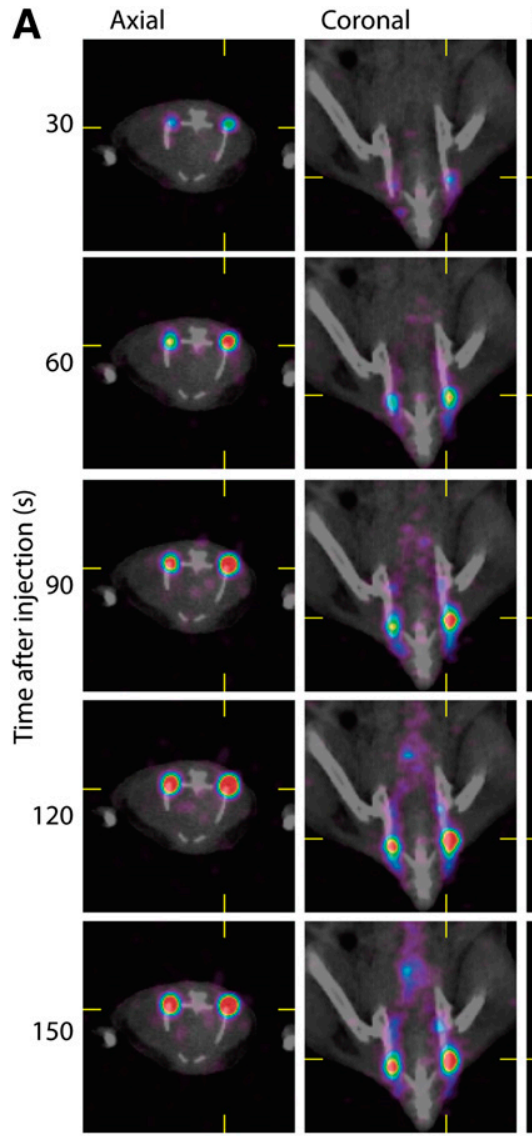

50
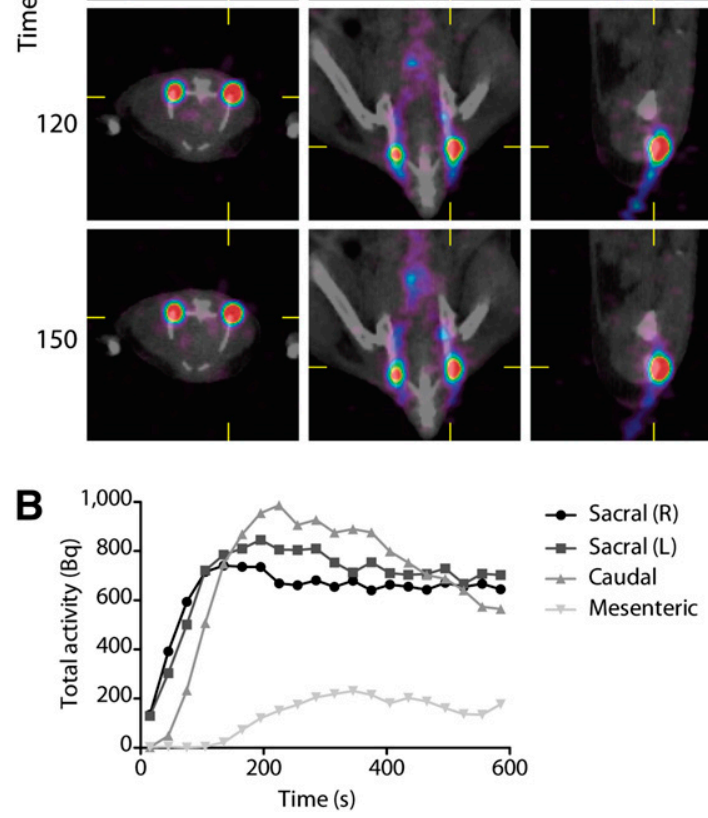

FIGURE 3. Quantitative and dynamic imaging of lymphatic ${ }^{18} \mathrm{~F}-$ FDG transport. (A) Axial, coronal, and sagittal views of dynamic imaging (30-s frames starting at time of injection) of ${ }^{18} \mathrm{~F}-\mathrm{FDG}$ transport in lymphatics. Acquisition of rapidity of flow through vessels and uptake into nodes, with both high spatial and longitudinal resolution, is an advantage of this technique. (B) PET information is inherently quantitative, providing activity concentration information. Here, regions of interest were drawn around LN to assess lymphatic function by computing uptake over time (over linear regions of curve). For right sacral, left sacral, caudal, and mesenteric nodes, this yields $6.47,6.57,5.67$, and $1.13 \mathrm{~Bq} / \mathrm{s}$, respectively.

further surgical exposure (PET acquisition is included as Supplemental Fig. 2). The resected node continues to generate $\mathrm{CR}$ after excision and therefore provides immediate verification of the lymphatic nature of removed tissue.

In comparison, CR imaging reveals that the retroorbital injection of ${ }^{18} \mathrm{~F}$-FDG results in accumulation in the kidneys and liver, rather than at any specific nodal sites. The sacral node identified using intradermal injection is not visible in the intravenously injected subject, even after the node is revealed by surgical incision. The optical approach enables detection of sites of radiotracer uptake and white-light anatomic localization using the same camera. Previously, ${ }^{99 \mathrm{~m} T c-S C}$ has been applied as a single tracer for both lymphoscintigraphy (or SPECT) and intraoperative guidance through use of operating-room $\gamma$-cameras and handheld $\gamma$-probes. However, adoption of these technologies has been limited by lack of anatomic reference. Intradermal ${ }^{18} \mathrm{~F}-\mathrm{FDG}$ provides high spatial resolution for optical imaging to guide resection of the small nodes in addition to the option of highresolution preoperative PET.

\section{Immunohistochemistry}

The excised tissues were validated as LNs using immunohistochemistry (Fig. 6D). The hematoxylin and eosin counterstain revealed the high cellular density indicative of lymphatic tissue. Podoplanin is a specific marker for lymphatic tissues (31-33), and staining for podoplanin confirms the presence of lymphatic endothelium in resected tissues.

\section{DISCUSSION}

Here, we have described the intradermal application of ${ }^{18} \mathrm{~F}-\mathrm{FDG}$ for the dual purposes of mapping the lymphatic vessels and nodes using PET and for Cerenkov-guided surgery. Systemic intravenous injection of ${ }^{18} \mathrm{~F}-\mathrm{FDG}$ for lymphographic examination has been explored in a host of studies. These studies often observe increased uptake at inflamed and invaded nodal sites. Clinically, the increased uptake indicates and helps identify disease. However, intravenous administration results in a substantial background signal, with renal clearance of the tracer required before any useful acquisition, abrogating the ability to conduct early-time-point or dynamic studies. In addition, imaging of nodes is difficult in regions of the pelvis (relevant for diseases such as prostate and cervical cancer) because of bladder uptake. The intradermal approach described here avoids the systemic and vascular distribution of the tracer at early time points, and the lymphatics are still clearly visible as late as $1 \mathrm{~h}$ after injection. This visibility allows for mapping of the lymphatic system directly through positron energy (Cerenkov) and annihilation photon (PET) events.

Intradermal injection of radiotracers leading to accumulation in the draining LN has previously been observed, either by accident or by design $(34,35)$. However, macromolecules or nanoparticles have been the preferred formulation for lymph-mapping agents in use or in development (36). The rationale has been that such materials will avoid diffusion into the vascular compartment and stay at a draining node.

This work shows that an approved small molecule is well suited to this purpose. In fact, this approach had been previously attempted (in the context of a biodistribution study) and dismissed, because only regional nodes could be detected (35). However, technologic advancements in the imaging sciences (namely the development of PET/CT, MRI/PET, greater-resolution PET, and Cerenkov imaging) now enable 


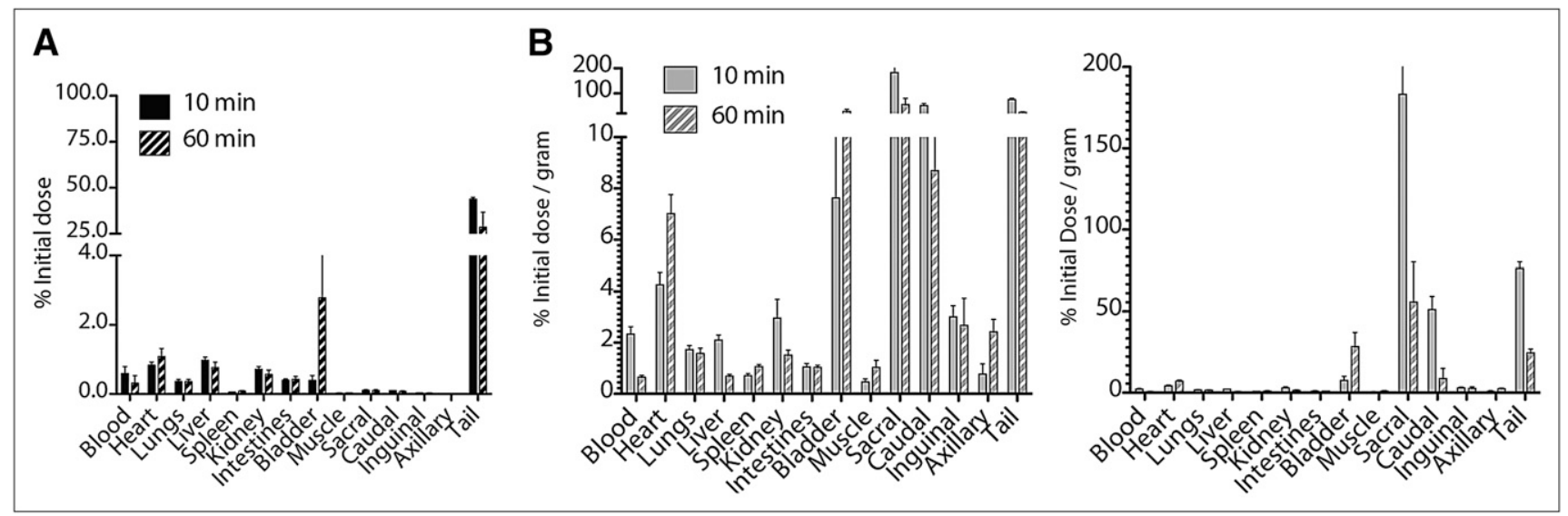

FIGURE 4. Biodistribution of intradermally injected small-molecule tracer. (A) Bar chart showing \%ID distribution of locally administered ${ }^{18} \mathrm{~F}-\mathrm{FDG}$ in major organs and LNs at 10 and $60 \mathrm{~min}$ after injection. Radiotracer slowly leaves dermal site of tail to circulation, renal excretion, and accumulation in bladder. (B) Normalizing dose distribution for mass of tissue (\%ID/g) demonstrates draining-lymph-specific transit of tracer. Retained radiotracer at injection site continues to be carried through lymphatics from injection site to draining nodes, enabling continued visualization. Left and right plots have different scales to show organ and nodal \%ID/g, respectively.

this type of imaging as a viable, powerful, and facile technique for efficient and sensitive lymphatic mapping localized with anatomic information. Our results demonstrate that ${ }^{18} \mathrm{~F}-\mathrm{FDG}$, the most widely used and readily available PET radiopharmaceutical, enables exquisite lymphography and has intraoperative potential through Cerenkov imaging.

On the basis of cost, effectiveness, and availability, ${ }^{99 \mathrm{~m}} \mathrm{Tc}-\mathrm{SC}$ is an excellent agent. However, intradermal administration of an ${ }^{18} \mathrm{~F}$-labeled small molecule results in several advantages. Initially, the tracer is confined to the lymphatic system compartment. As demonstrated with dynamic imaging, approximately $40 \mathrm{~min}$ are required for the tracer to become present in other tissues at comparative levels. The use of a positron emitter, ${ }^{18} \mathrm{~F}$, provides high-resolution tomographic and quantitative distribution information. These features, in conjunction with the anatomic localization provided by CT, result in finely detailed images of the lymphatic system. ${ }^{18} \mathrm{~F}$-FDG also has favorable distribution characteristics. Unlike ${ }^{99 \mathrm{~m}} \mathrm{Tc}-\mathrm{SC}$, the small-molecule radioglucose clears from the injection site fairly quickly, thus avoiding the obscuring of features at or near the injection.
A clear advantage of the small-molecule approach for patient management is that draining nodes located outside the immediate (or for prostate, routinely dissected) area can be tomographically localized by PET (37). The resolution and sensitivity of PET enable the discernment of both the lymphatic vessels and the LN with great detail in deep tissue. This ability has important implications for preclinical investigation. Despite its importance, the lymphatic system remains poorly understood in part because of a lack of imaging tools (38). The positron lymphography approach presented here can aid analysis of rodent models (i.e., that possess lymphatic structures many times smaller than those of primates) and applies to LN drainage from appendages such as the tail (Figs. 1 and 2) and feet (Supplemental Fig. 5). The LNs as visualized by PET appear larger than they are, because the dimensions of these structures are well below the resolution (full width at half maximum) of small-animal PET. Lymphoscintigraphy and SPECT with ${ }^{99 \mathrm{~m}} \mathrm{Tc}-\mathrm{SC}$ have relatively poorer resolving power, and near-infrared approaches have limited depth penetration in contrast to the whole-body method using ${ }^{18} \mathrm{~F}-\mathrm{FDG}$.

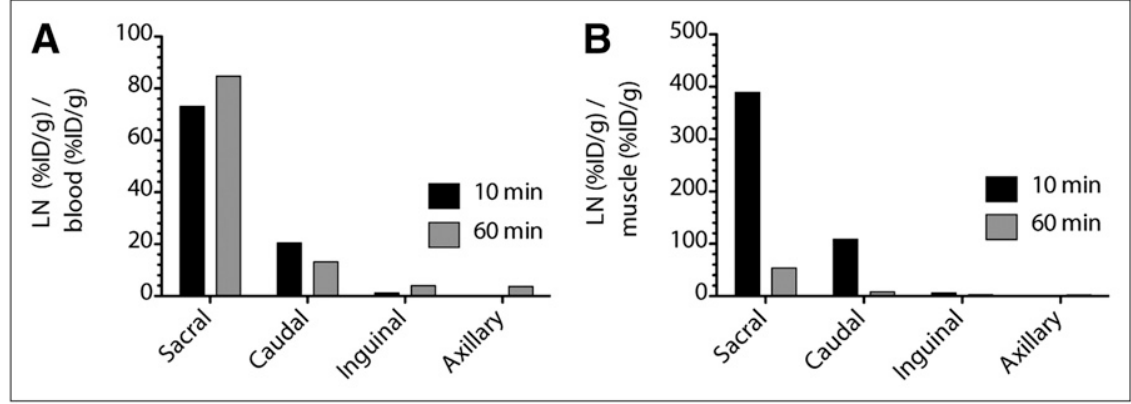
lary) uptake is low because these nodes are not within drainage of injection site, which drains to deep pelvic nodes
FIGURE 5. Contrast ratio of ${ }^{18} \mathrm{~F}-\mathrm{FDG}$ to blood and muscle. (A) Ratios of \%ID/g from draining $\mathrm{LN}$ and blood are high, affording clear distinction between lymphatics and surrounding tissues. (B) Contrast derived from $\% \mathrm{ID} / \mathrm{g}$ between $\mathrm{LN}$ and muscle enables clear delineation of nodes. LNs not in the draining path from the injection site (inguinal and axillary) do not produce significant ratios with respect to background. From 10 to $60 \mathrm{~min}$, ratio of $\mathrm{LN}$ to muscle drops as conscious and ambulatory mice increase muscle uptake. Inguinal (and axil- 

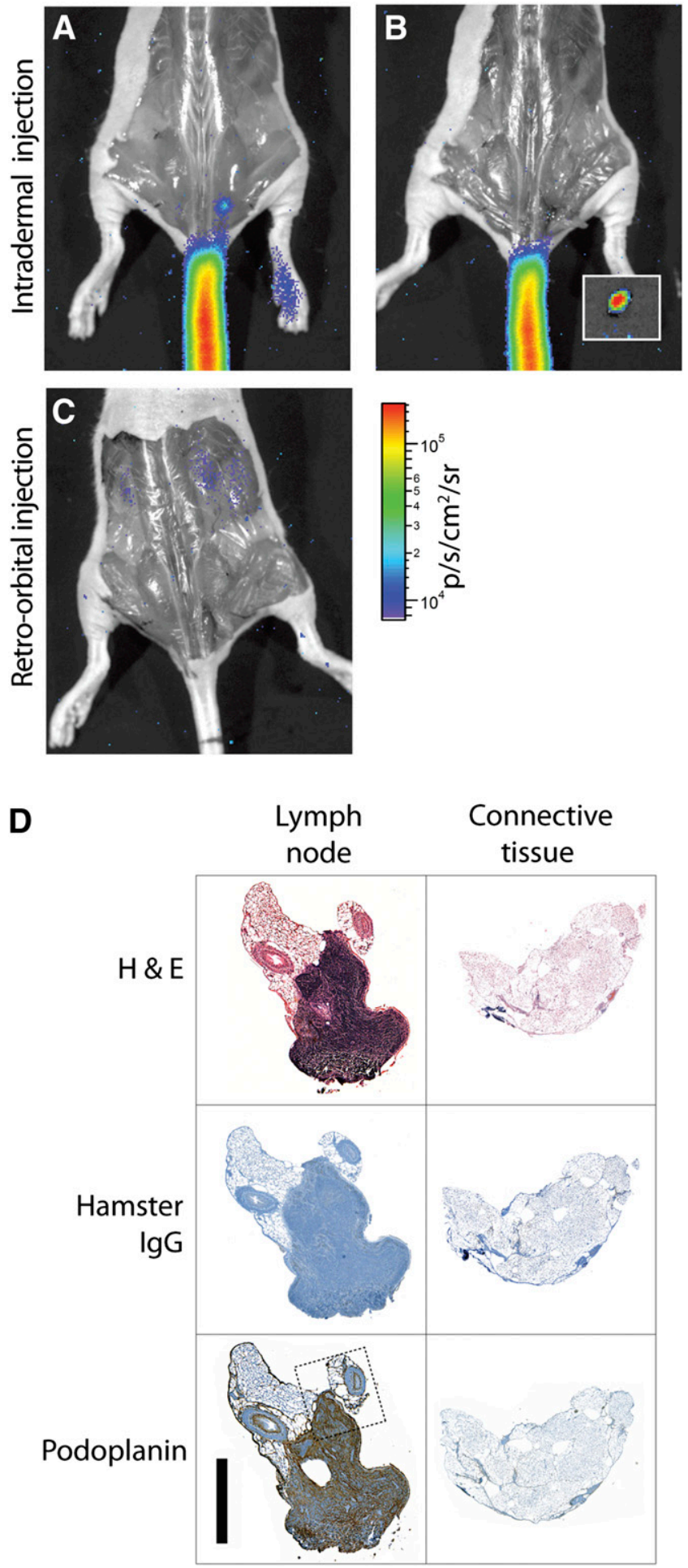

FIGURE 6. Cerenkov-guided surgical resection of ${ }^{18} \mathrm{~F}-\mathrm{FDG}-$ bearing LN, 10 min after injection, with surgical validation. (A) Lateral-tail intradermal injection yields greater uptake in 1 sacral node, seen with just skin removed. PET image is included as Supplemental Figure 2. (B) CR guides resection of node, magnified in inset. (C) Systemic administration of ${ }^{18} \mathrm{~F}-\mathrm{FDG}$ (retroorbital injection) does not enable identification of nodes, even after surgical exposure. Signal from renal clearance can be seen. (D) Immunohistologic verification of Cerenkov-guided excised tissues. Resected node as indicated by $\mathrm{CR}$, along with surrounding connective tissue, was stained with
Positron decay produces CR that is detectable by sensitive cameras. Cerenkov-guided surgical resection of LNs would be an advance over current techniques in that it requires the administration of a single, nontoxic agent to localize nodes. ${ }^{99 \mathrm{~m}} \mathrm{Tc}$ is a $\gamma$-emitter with virtually no positron emission and does not generate $\mathrm{CR}$.

$\mathrm{CR}$ from nodal uptake of ${ }^{18} \mathrm{~F}-\mathrm{FDG}$ was used to guide resection of sacral nodes that are virtually undetectable without alternative contrast (vital dyes). However, the use of dyes (isosulfan blue) is hampered by washout from the node of interest and potential allergic reaction (39). As seen from the dynamic imaging (Fig. 2), the transit of ${ }^{18}$ F-FDG to LNs with minimal background is sustained over a sufficiently long period (at least $1 \mathrm{~h}$ ) to potentially facilitate intraoperative events and the short half-life, ${ }^{18} \mathrm{~F}-\mathrm{FDG}$ can be repeatedly administered for lymphatic delineation in a research or surgical lymph mapping study.

\section{CONCLUSION}

The lymphatic vasculature plays a critical role in maintaining the body's fluid balance. Aberrant lymph function and lymphadenopathy are significant indicators of the presence and extent of disease. Approaches for visualizing these events include ${ }^{99 \mathrm{~m} T c-S C}$ lymphoscintigraphy, SPECT, absorbent optical imaging, or albumin-bound near-infrared fluorescence imaging (16). However, scintigraphic and SPECT approaches have limited resolution and anatomic localization, whereas fluorescent studies are nonquantitative, tissue depth-dependent, and hampered by a limited choice of approved fluorophores (namely, indocyanine green). The ability to image the lymphatics tomographically at the detail of its fine structure, with high-sensitivity and in a quantitative regime, may greatly enhance the study of these systems.

We have shown that the common PET tracer ${ }^{18} \mathrm{~F}-\mathrm{FDG}$ is localized in the LNs draining from an intradermal injection. The high sensitivity and low background provide excellent discrimination for localizing LNs and studying lymphatic vessel transport. Dynamic imaging can be performed with this easily obtained and approved tracer. Importantly, through imaging developments to capture CR from positron emissions, it is also possible to optically detect nodal uptake of this radiotracer in vivo, enabling dual-modality imaging of an approved tracer for preoperative tomographic whole-body imaging along with intraoperative planar anatomic guidance using CR, $\gamma$-probes, and positron detectors. We suggest that the use of a widely used PET tracer, through an alternative delivery mechanism, presents an important preclinical and clinical advance toward greater understanding of and care for diseases involving the lymphatic system.

hematoxylin and eosin, control hamster lgG, and podoplanin. The stromal region of node is indicated by dense hematoxylin and eosin and positive podoplanin staining. Dotted line indicates region of magnification (included as Supplemental Fig. 4). Scale bar, $1 \mathrm{~mm}$. $\mathrm{H} \& \mathrm{E}=$ hematoxylin and eosin. 


\section{DISCLOSURE STATEMENT}

The costs of publication of this article were defrayed in part by the payment of page charges. Therefore, and solely to indicate this fact, this article is hereby marked "advertisement" in accordance with 18 USC section 1734.

\section{ACKNOWLEDGMENTS}

We thank the staff of the Radiopharmacy at MSKCC. We give special thanks to Dr. Steve Larson for his input and Drs. Bill Strauss, Michael McDevitt, Jason Lewis, Kishore Pillarsety, and Mesruh Turkekul. This study was supported in part through the R25T Molecular Imaging Fellowship: Molecular Imaging Training in Oncology (5R25CA09694507); the 2011 SNM Postdoctoral Molecular Imaging Scholar Program; the Louis V. Gerstner Young Investigator Award; ARRA 73950; NIH grants 1R01EB014944-01, R24 CA83084, and P30 CA08748; and the MCCF. No other potential conflict of interest relevant to this article was reported.

\section{REFERENCES}

1. Alitalo K, Tammela T, Petrova TV. Lymphangiogenesis in development and human disease. Nature. 2005;438:946-953.

2. Lucey BC, Stuhlfaut JW, Soto JA. Mesenteric lymph nodes seen at imaging: causes and significance. Radiographics. 2005;25:351-365.

3. Balch CM, Buzaid AC, Soong S-J, et al. Final version of the American Joint Committee on Cancer Staging System for cutaneous melanoma. J Clin Oncol. 2001;19:3635-3648

4. Gershenwald JE, Thompson W, Mansfield PF, et al. Multi-institutional melanoma lymphatic mapping experience: the prognostic value of sentinel lymph node status in 612 stage I or II melanoma patients. J Clin Oncol. 1999;17:976-983.

5. Pagliarulo V, Hawes D, Brands FH, et al. Detection of occult lymph node metastases in locally advanced node-negative prostate cancer. J Clin Oncol. 2006;24: 2735-2742.

6. Tinga DJ, Timmer PR, Bouma J, Aalders JG. Prognostic significance of single versus multiple lymph node metastases in cervical carcinoma stage IB. Gynecol Oncol. 1990;39:175-180.

7. Delgado G, Bundy B, Zaino R, Sevin B-U, Creasman WT, Major F. Prospective surgical-pathological study of disease-free interval in patients with stage ib squamous cell carcinoma of the cervix: a gynecologic oncology group study. Gynecol Oncol. 1990;38:352-357.

8. McMasters KM, Tuttle TM, Carlson DJ, et al. Sentinel lymph node biopsy for breast cancer: a suitable alternative to routine axillary dissection in multi-institutional practice when optimal technique is used. J Clin Oncol. 2000;18: 2560-2566.

9. Fisher ER, Costantino J, Fisher B, et al. Pathologic findings from the National Surgical Adjuvant Breast Project (NSABP) protocol B-17: five-year observations concerning lobular carcinoma in situ. Cancer. 1996;78:1403-1416.

10. Carter CL, Allen C, Henson DE. Relation of tumor size, lymph-node status, and survival in 24,740 breast-cancer cases. Cancer. 1989;63:181-187.

11. Harisinghani MG, Barentsz J, Hahn PF, et al. Noninvasive detection of clinically occult lymph-node metastases in prostate cancer. N Engl J Med. 2003;348:2491-2499.

12. Larson SM, Nelp WB. Radiopharmacology of a simplified technetium-99m-colloid preparation for photoscanning. J Nucl Med. 1966;7:817-826.

13. Mortimer PS, Simmonds R, Rezvani M, Robbins M, Hopewell JW, Ryan TJ. The measurement of skin lymph flow by isotope clearance; reliability, reproducibility, injection dynamics, and the effect of massage. J Invest Dermatol. 1990;95:677-682.
14. Showalter TN, Miller TR, Huettner P, Rader J, Grigsby PW. ${ }^{18}$ F-fluorodeoxyglucosepositron emission tomography and pathologic tumor size in early-stage invasive cervical cancer. Int J Gynecol Cancer. 2009;19:1412-1414.

15. Hirsch J, Tisnado J. Cho, Beachley M. Use of isosulfan blue for identification of lymphatic vessels: Experimental and clinical evaluation. AJR. 1982;139:10611064.

16. Sevick-Muraca EM. Translation of near-infrared fluorescence imaging technologies: emerging clinical applications. Annu Rev Med. 2012;63:217-231.

17. Tsien RY. Imagining imaging's future. Nat Rev Mol Cell Biol. 2003;(suppl): SS16-SS21.

18. Heuveling DA, Visser GWM, Baclayon M, et al. ${ }^{89} \mathrm{Zr}$-nanocolloidal albuminbased PET/CT lymphoscintigraphy for sentinel node detection in head and neck cancer: preclinical results. J Nucl Med. 2011;52:1580-1584.

19. Ballou B, Ernst LA, Andreko S, et al. Sentinel lymph node imaging using quantum dots in mouse tumor models. Bioconjug Chem. 2007;18:389-396.

20. Clark WH, Mihm MC Jr, Reed RJ, Ainsworth AM. The lymphocytic infiltrates of the skin. Hum Pathol. 1974;5:25-43.

21. Robertson R, Germanos MS, Li C, Mitchell GS, Cherry SR, Silva MD. Optical imaging of Cerenkov light generation from positron-emitting radiotracers. Phys Med Biol. 2009;54:N355-N365.

22. Ruggiero A, Holland JP, Lewis JS, Grimm J. Cerenkov luminescence imaging of medical isotopes. J Nucl Med. 2010;51:1123-1130.

23. Holland JP, Normand G, Ruggiero A, Lewis JS, Grimm J. Intraoperative imaging of positron emission tomographic radiotracers using Cerenkov luminescence emissions. Mol Imaging. 2011;10:177-186.

24. Guide for the Care and Use of Laboratory Animals. Washington, DC: National Academy Press; 1996.

25. Beattie BJ, Forster GJ, Govantes R, et al. Multimodality registration without a dedicated multimodality scanner. Mol Imaging. 2007;6:108-120.

26. Qi J, Leahy RM, Cherry SR, Chatziioannou A, Farquhar TH. High-resolution 3D Bayesian image reconstruction using the micropet small-animal scanner. Phys Med Biol. 1998;43:1001-1013.

27. Beattie BJ, Thorek DL, Schmidtlein CR, Pentlow KS, Humm JL, Hielscher AH. Quantitative modeling of Cerenkov light production efficiency from medical radionuclides. PLOS ONE. 2012;7:e31402.

28. Boardman KC, Swartz MA. Interstitial flow as a guide for lymphangiogenesis. Circ Res. 2003;92:801-808.

29. Clavin NW, Avraham T, Fernandez J, et al. TGF-b1 is a negative regulator of lymphatic regeneration during wound repair. Am J Physiol Heart Circ Physiol. 2008;295:H2113-H2127

30. Yan A, Avraham T, Zampell JC, Aschen SZ, Mehrara BJ. Mechanisms of lymphatic regeneration after tissue transfer. PLOS ONE. 2011;6:e17201.

31. Kriehuber E, Breiteneder-Geleff S, Groeger M, et al. Isolation and characterization of dermal lymphatic and blood endothelial cells reveal stable and functionally specialized cell lineages. J Exp Med. 2001;194:797-808.

32. Matsui K, Breitender-Geleff S, Soleiman A, Kowalski H, Kerjaschki D. Podoplanin, a novel 43-kDa membrane protein, controls the shape of podocytes. Nephrol Dial Transplant. 1999;14(suppl 1):9-11.

33. Ordóñez NG. Podoplanin: a novel diagnostic immunohistochemical marker. $A d v$ Anat Pathol. 2006;13:83-88.

34. Schillaci O, Calabria F, Tavolozza M, et al. ${ }^{18} \mathrm{~F}$-choline PET/CT physiological distribution and pitfalls in image interpretation: experience in 80 patients with prostate cancer. Nucl Med Commun. 2010;31:39-45.

35. Wahl RL, Kaminski MS, Ethier SP, Hutchins GD. The potential of 2-deoxy-2 [18f]fluoro-D-glucose (FDG) for the detection of tumor involvement in lymph nodes. J Nucl Med. 1990;31:1831-1835.

36. Wilhelm AJ, Mijnhout GS, Franssen EJ. Radiopharmaceuticals in sentinel lymphnode detection: an overview. Eur J Nucl Med. 1999;26(4, suppl):S36-S42.

37. Vermeeren L, Valdés Olmos RA, Meinhardt W, et al. Value of SPECT/CT for detection and anatomic localization of sentinel lymph nodes before laparoscopic sentinel node lymphadenectomy in prostate carcinoma. J Nucl Med. 2009;50: 865-870.

38. Servais EL, Colovos C, Bograd AJ, White J, Sadelain M, Adusumilli PS. Animal models and molecular imaging tools to investigate lymph node metastases. $\mathrm{J} \mathrm{Mol}$ Medm (Berl). 2011;89:753-769.

39. Albo D, Wayne JD, Hunt KK, et al. Anaphylactic reactions to isosulfan blue dye during sentinel lymph node biopsy for breast cancer. Am J Surg. 2001;182:393-398. 